\title{
Common region wins the competition between extrinsic grouping cues: Evidence from a task without explicit attention to grouping
}

\author{
Pedro R. Montoro ${ }^{1}$. Cristina Villalba-García ${ }^{2,3}$ • Dolores Luna ${ }^{1}$. José A. Hinojosa $a^{2,3}$
}

Published online: 1 March 2017

(C) Psychonomic Society, Inc. 2017

\begin{abstract}
The competition between perceptual grouping factors is a relatively ignored topic, especially in the case of extrinsic grouping cues (e.g., common region or connectedness). Recent studies have examined the integration of extrinsic cues using tasks that induce selective attention to groups based on different grouping cues. However, this procedure could generate alternative strategies for task performance, which are non-related to the perceptual grouping operations. In the current work, we used an indirect task, i.e. repetition discrimination task, without explicit attention to grouping cues to further examine the rules that govern dominance between competing extrinsic grouping factors. This procedure allowed us to obtain an unbiased measure of the competition between common region and connectedness cues acting within the same display. The results corroborate previous data showing that grouping by common region dominated the perceived organization of the display, even though the phenomenological strength of the grouping cues was equated for each participant by means of a preliminary scaling task. Our results highlight the relevance of using indirect tasks as an
\end{abstract}

Electronic supplementary material The online version of this article (doi:10.3758/s13423-017-1254-3) contains supplementary material, which is available to authorized users.

Pedro R. Montoro

prmontoro@psi.uned.es

1 Departamento de Psicología Básica 1, Facultad de Psicología. UNED, C/Juan del Rosal 10, 28040 Madrid, Spain

2 Instituto Pluridisciplinar, Universidad Complutense de Madrid, Madrid, Spain

3 Departamento de Psicología Básica I, Universidad Complutense de Madrid, Madrid, Spain essential tool for the systematic study of the integration of extrinsic grouping cues.

Keywords Gestalt $\cdot$ Perceptual grouping $\cdot$ Extrinsic principles · Grouping by common region · Grouping by connectedness

\section{Introduction}

Perceptual organization is the architecture studio of vision, which is in charge of fitting together the pieces of the retinal mosaic to build a tidy visual experience. The seminal work of Wertheimer (1923) described the main principles of perceptual grouping that determine what regions of an image constitute perceptual units or objects (Brooks, 2015), such as proximity, similarity, common fate, symmetry, parallelism, closure or good continuation. From the proposal of Palmer (1992, 1999), these classic Gestalt laws are classified as intrinsic grouping cues since they are based on built-in properties of the discrete elements (e.g., their shape, position), and a new set of extrinsic grouping principles which was added based on relationships between the discrete elements and other external elements that induce them to group. For example, grouping by common region (Palmer, 1992) refers to the fact that elements located within the same bounded region tend to be grouped together. Also, grouping by connectedness is the tendency of elements that are connected by other elements to be seen as part of the same group (Palmer \& Rock, 1994). Prior evidence indicates that these principles can compete effectively against some of the classic Gestalt principles (Brooks, 2015). Remarkably, the ecological rationale behind connectedness arises from the fact that several parts of an object are frequently connected to one another despite showing a visual disparity in color or form (Brooks, 2015). Common region also seems 
to have an ecological basis arising from hierarchically embedded parts (e.g., leopard's spots or features of a face; Palmer, 1992). Indeed, effects of these grouping cues on object perception and behavior have been reported. For instance, common region can be particularly effective at communicating complex informational structures in realistic environments (Bae \& Watson, 2014). Also, element connectedness can affect object perception in patients with neurological lesions altering visual processing (Gillebert \& Humphreys, 2015). Furthermore, the relevance of the extrinsic cues for behavior have been persuasively shown by several studies reporting that very young infants (3- 4 months old) are sensitive to the connectedness and common region principles (Quinn \& Bhatt, 2015).

A well-known limitation of the grouping principles is that they are under ceteris paribus rules, so that they can predict the configuration perceived only while "other things remain equal" (Palmer, 1999). This restriction makes it difficult to predict the combined effects of multiple conflicting factors applied to the same display. In an attempt to solve this deficiency, several studies have examined the integration of multiple intrinsic Gestalt principles when they are combined (e.g., proximity, similarity, collinearity, etc). The main findings on this issue are compatible with additive effects of grouping factors and support the independence of the respective intrinsic cues (see Kubovy \& van den Berg, 2008 or Peterson \& Kimchi, 2015, for a review). The combined grouping effect of extrinsic cues has only recently been considered in three studies (Luna and Montoro, 2011; Montoro and Luna, $2015 \mathrm{a}, \mathrm{b})$ that examined cooperation and competition between intrinsic principles of proximity and similarity and the extrinsic principle of common region by means of a phenomenological method to quantify spontaneous grouping (Quinlan and Wilton, 1998). The results of these studies showed that conflicting cues made grouping weaker and unstable, while cooperating cues made grouping stronger and steady, in line with an additive model for the combination of grouping factors, as proposed by Kubovy and van den Berg (2008).

An important theoretical problem concerns the dominance of extrinsic grouping principles when they operate simultaneously in the same display and compete. Findings on this issue might also have some practical consequences such as their potential application for designers to communicate complex informational structures (Bae \& Watson, 2014). Thus, a crucial issue in the study of perceptual grouping is the identification of the rules or conditions that govern which principle is dominant when two (or more) principles compete (Peterson \& Kimchi, 2015). In this line, Luna and collaborators (2016) examined competition between intrinsic and extrinsic grouping cues by inducing their participants to perceive the displays grouped in a determined way before responding, which allowed the measurement of the accuracy and the speed of responses. This scheme provided measures of perceptual grouping dominance similar to those collected in research concerning dominance processing in hierarchical stimuli (Navon, 1977). In Luna et al.'s Experiment 2, a dominance of the common region cue over connectedness was indicated by (1) an advantage effect of common region (faster responses to groups formed by common region than to those formed by connectedness) and (2) a bidirectional but asymmetrical interference due to the fact that grouping by connectedness was slowed by the presence of the competing common region cue more than vice versa. Similarly, a dominance of common region over shape similarity was found in Experiment 3. Notably, in both experiments, the strength of the grouping cues was previously equated with phenomenological reports.

Crucially, the use of tasks using selective attention to groups to study extrinsic grouping factors could be particularly threatened by the intrusion of alternative strategies of performing the task, which are non-related to the perceptual grouping operations (Palmer \& Beck, 2007). In this sense, it seems possible that some participants attend to the inductors (i.e. contours defining a region or lines connecting items) to achieve task requirements while ignoring the discrete elements to be grouped. For instance, in Luna et al. (2016), some participants might have responded by considering only the location of the extrinsic inductors on either the right or the left.

Given the importance of identifying the conditions that determine which of two competing extrinsic principles will dominate the perceived organization, in the present work we further examined the competition between common region and element connectedness principles overcoming prior limitations. In addition, an important issue was to explore the dominance dynamics of extrinsic grouping cues in tasks which did not require selective attention to the grouping cues in order to examine whether the pattern of results is similar to that found using approaches demanding attention to grouping (Luna et al., 2016).

To investigate the competition between common region and connectedness, we used the repetition discrimination task (RDT; Palmer \& Beck, 2007), an indirect task that does not require explicit attention to grouping cues. This task also allowed the examining and preventing of strategic effects that might be found in selective attentions tasks (Palmer \& Beck, 2007). This task provides an unbiased measure of the dominance dynamics of extrinsic grouping based on reactions times (RTs) and accuracy. In particular, the participants had to discriminate the repeated shape (square or circle) in a row of nine alternating elements. The grouping factors could influence the perceived organization of squares and circles by facilitating or hindering the task. Thus, RT differences between experimental conditions could be taken as an indirect measure of grouping effects. Remarkably, the RDT has been successfully employed to provide indirect evidence for both common region and connectedness single cues (Palmer \& Beck, 2007, Experiment 3). However, to our knowledge, the RDT has 
never been used to study the competition of two different grouping factors acting in the same display. A critical factor to consider when combining two grouping cues is their grouping strength, that is, the subjective saliency of the respective grouping factors (Schmidt \& Schmidt, 2013). Thus, we first conducted a scaling task to select the appropriate stimuli in order to ensure that the grouping strength of both cues was equated, as recommended in Kubovy and van den Berg (2008). Since prior studies observed between-subjects divergences in phenomenological measures of grouping (e.g., Schmidt \& Schmidt, 2013; Montoro \& Luna, 2015a, b), we obtained individual ratings of grouping strength for every participant from the scaling task. This allowed us to personalize the stimulus set displayed in the subsequent RDT. In the scaling task, participants had to equate the grouping strength of both principles by adjusting the thickness of the connectors acting as connectedness cues while the physical features of the common region inductor remained unaltered. We chose a simple one-dimension scaling task to make it more intuitive to participants.

\section{Method}

\section{Participants}

Twelve undergraduate students (five men; age range: 1946 years, mean $=27.3, \mathrm{SD}=8.6$ ) from the UNED participated in the experiment and received course credits for their participation. All of them had normal or corrected-to-normal vision. The experimental procedure was approved by the Local Ethics Committee and conforms to the Declaration of Helsinki.

\section{Apparatus}

The stimuli were displayed on a 19-inch (c.48.25-cm) LCDLED color monitor with a $75-\mathrm{Hz}$ refresh rate, a 5:4 aspect ratio, and a resolution of $1280 \times 1024$ pixels, controlled by a personal computer running E-Prime 2.0 software (Psychology Software Tools, 1996-2013). Viewing distance was approximately $60 \mathrm{~cm}$. Responses were recorded via a standard keyboard.

\section{Stimuli}

The stimulus set was based on Palmer and Beck's (2007) displays. The basic display consists of a row of nine dark (RGB: $0 ; 0.06 \mathrm{~cd} / \mathrm{m}^{2}$ ) equidistant elements that alternate between squares and circles, which subtended $9 \times 9 \mathrm{~mm}$ $\left(0.86^{\circ}\right.$ v.a. $)$. The entire array of nine elements measured $152 \mathrm{~mm}\left(14.22^{\circ}\right.$ v.a. $)$ vertically. The edge-to-edge distance between elements was $9 \mathrm{~mm}\left(0.86^{\circ}\right.$ v.a. $)$.

For the RDT, 48 different stimuli were drawn (see Fig. 1b), 24 for the single conditions and 24 displays for the competing conditions. The nine elements alternated between squares and circles, except for a single pair of adjacent similar shapes somewhere within the middle seven elements. In the common region-only condition, four light gray (RGB: $128 ; 14.1 \mathrm{~cd} / \mathrm{m}^{2}$ ) oval outlines $\left(33 \times 21 \mathrm{~mm} ; 3.15^{\circ} \times 2.01^{\circ}\right.$ v.a. $)$ and a semi-oval were added to the pattern as common region cues. In the connectedness-only condition, five light gray (RGB: 128; $14.1 \mathrm{~cd} / \mathrm{m}^{2}$ ) connectors (vertical length: $9 \mathrm{~mm} ; 0.86^{\circ}$ v.a.) were added as connectedness cues. The thickness value of the connectors was personalized for each participant based on the results of the scaling task (see Results section). In the competing conditions, both ovals and connectors were included as part of the stimuli.

In the scaling task, all the stimuli were competing patterns alternating square/circle shapes with absent target pairs (i.e. without a repeated pair of elements; see Fig. 1a). Thirty different stimuli were created by manipulating the thickness value of the connectors from 1 to 30 pixels by one-unit increments (pixels: $1,2 \ldots, 29,30$ ).

\section{Design and procedure}

The $2 \times 2$ design included two within-subjects factors: stimulus type (single or competing grouping cues) and grouping cue joining the target pair (common region vs. connectedness). These four different types of stimuli were combined with six
SCALING TASK

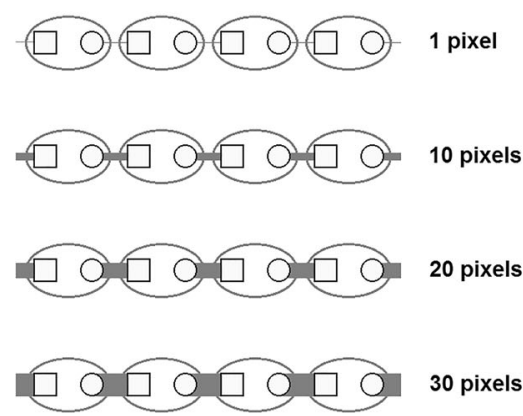

REPETITION DISCRIMINATION TASK

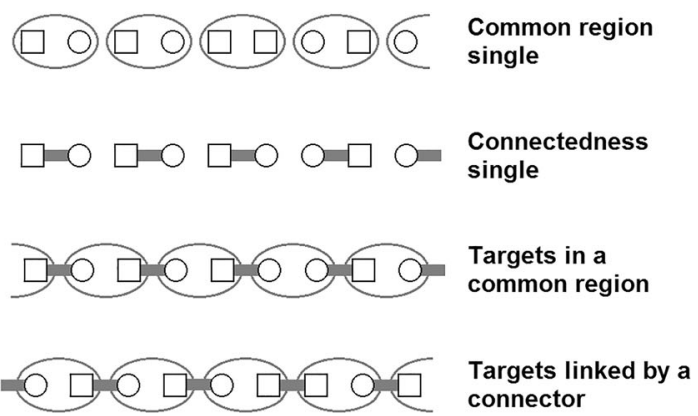

Fig. 1 Examples of some stimuli displayed in the scaling task and in the RDT. The thickness of the connectors in the right panel is equal to 15 pixels 
different positions of the target pair (elements $2-3,3-4,4-5$, $5-6,6-7,7-8$, counting from the left), two shapes of the target pair (squares vs. circles) and two repetitions to obtain 96 trials in each experimental block. RTs and error rates were measured.

Participants were tested individually in a dimly lit room in two different sessions, one for the scaling task, and another one for the RDT. In the scaling task, participants had to adjust the thickness of the connectors joining elements until their grouping strength appeared as equally strong as the grouping strength of the common region cue, so that the visual grouping of the elements in pairs were equally salient in figures joined together by connectors and in figures included in ovals. In order to avoid confounds, the instructions emphasized that judgments must be based on the grouping strength or subjective salience of the extrinsic cues, which does not necessarily imply that the inductors (connectors and ovals) had to be equated in physical thickness. Each participant completed one practice trial and six scaling trials: three trials starting with 1 pixel in thickness and other three beginning with 30 pixels in thickness. The order of presentation of the increasing and decreasing trials was alternated between subjects. Participants increased or decreased the thickness of the connectors by pressing the right or the left arrow keys of the keyboard with the index fingers of both hands. After verbal confirmation of their decision, the specific thickness value was recorded without informing the participant. There was no time limit. A mean pixels value collapsing the six judgments was computed for each participant, which was implemented as the thickness value of the connectors in the stimuli included in the subsequent RDT.

In the RDT, participants responded as quickly as possible while avoiding errors by pressing one of two keys (i.e. "Z" and " $M$ ") with their left or right index to indicate the shape (circle or square) of the repeated pair of elements. Key assignment was counterbalanced across participants. The stimulus array was presented on the center of the screen and remained until response. The inter-trial interval was $800 \mathrm{~ms}$. There were a practice block with 48 trials and six experimental blocks consisting of 96 trials each, for a total of 576 experimental trials. Feedback was provided only for the practice trials.

\section{Results}

\section{Scaling task}

Figure 2a shows the results for each participant The range of mean pixels adjusted values fluctuated between 7 and 18 $($ mean $=12 ; \mathrm{SD}=4.1)$, showing a remarkable variability among participants in their perception of relative grouping strength, as expected. In contrast, the rather small standard errors (see Fig. 2a) suggest that participants were very consistent in their judgments across the trials.

\section{Repetition discrimination task}

Median RTs of correct responses and mean accuracy rates were submitted to separate analyses of variance (ANOVAs) with stimulus type and grouping cue joining the target as within-subjects factors.

RT analysis Inaccurate responses (103 of 6,$912 ; 1.5 \%$ of trials) and RTs greater than 4000 or less than $200 \mathrm{~ms}$ (11 of 6,$809 ; 0.16$ of trials) were excluded from the RT analysis. Analyses on RTs showed a significant main effect of stimulus type, $F(1,11)=162.9, \mathrm{MSE}=6136, p<.001, \eta_{\mathrm{p}}{ }^{2}=.94$, indicating that RTs for single trials $(721 \mathrm{~ms})$ were shorter than those for competing ones $(1010 \mathrm{~ms})$. The main effect of Grouping cue was also significant, $F(1,11)=126.8$, MSE $=$ $8276.1, p<.001, \eta_{\mathrm{p}}{ }^{2}=.92$, showing that targets included in common region $(717 \mathrm{~ms}$ ) were responded to faster than targets joined by connectors (1013 ms). Finally, the interaction between the two factors was significant $F(2,30)=87.41$, MSE $=$
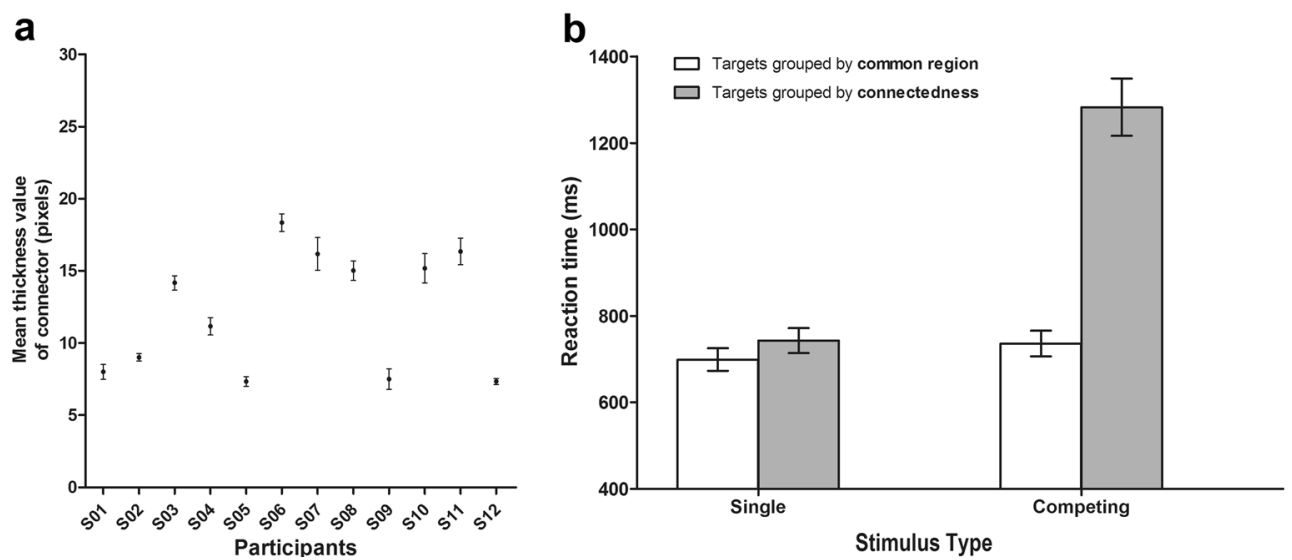

Fig. 2 a Mean adjusted thickness values for each participant in the scaling task. b Mean reaction times (ms) and standard error bars for the experimental conditions of the RDT 
$8709, p<.001, \eta_{\mathrm{p}}{ }^{2}=.89$, indicating that the interference effect of common region on the discrimination of targets grouped by connectedness (the difference between single and competing stimuli) was much greater ( $\Delta 540 \mathrm{~ms}: 1283-743 \mathrm{~ms})$ than the interference of connectedness on the discrimination of targets grouped by common region ( $\Delta 37 \mathrm{~ms}: 736-699 \mathrm{~ms}$ ). Pair-wise comparisons applying the Bonferroni correction showed significant differences between all pairs from the experimental conditions (all $p \mathrm{~s} \leq .01$ ).

Additionally, correlation analyses between the mean thickness value (pixels) of the connectors from the scaling task and the median RTs in the three conditions that displayed connectedness cues were conducted. None of the correlations reached significance (all $p \mathrm{~s}>.10$ ), supporting the independence between subjective grouping strength and the responses in the RDT.

Accuracy analysis Hit rates oscillated between $96 \%$ and $100 \%$. The results of the ANOVAs showed no significant main or interactive effects.

\section{Discussion}

In this study, we explored the integration of two competing extrinsic grouping cues by means of an indirect task without explicit attention to the grouping factors, which prevents strategic effects or biases unrelated to grouping operations. The results clearly supported that grouping by common region dominates the perceived organization of the display when the grouping factors were not explicitly attended. For the competing stimuli, the responses were considerably faster when the target elements were within the same oval compared to those observed in displays in which they remained in different ovals. The single conditions also showed faster responses for targets grouped by common region than for targets grouped by connectedness. Notably, RT differences between single cue conditions $(\Delta 37 \mathrm{~ms})$ were clearly smaller than those found between the competitive conditions ( $\Delta 540 \mathrm{~ms})$. Thus, even though both results reflect the dominance of common region over connectedness, it seems unlikely to attribute the rather large difference between competition conditions only to the existence of the small processing advantage found in the single conditions. Additionally, an influence of connectedness in conflicting conditions was observed, since RTs to target pairs linked by a connector in different ovals were significantly slower than in the common region single condition $(\Delta 7 \mathrm{~ms})$.

The relative dominance of common region over other grouping factors corroborated previous data obtained with direct measures based on phenomenological reports or choicereaction time tasks. In Luna and Montoro (2011), the common region cue was rated by observers as being stronger than proximity in a competing condition, even though both factors were judged as comparable in their respective single conditions (but see Montoro and Luna, 2015b). Also, Montoro et al. (2015) observed faster responses to common region than to luminance similarity in a direct choice RT task that did not include a competing condition. Similarly, using a task which required selective attention to the grouping cues, Luna et al. (2016) reported a dominance of common region over shape similarity and connectedness when they compete in the same display despite being equal in phenomenological grouping strength. Remarkably, the present results provide evidence for the first time of dominance for common region cues when they compete with connectedness in an indirect task that does not require explicit attention to grouping cues and avoids the use of some strategic biases. From an applied perspective, Bae and Watson (2014) observed that common region was the most accurate, fastest and most preferred structural communication in comparison to proximity, color similarity, connectedness and good continuation. Consequently, the authors recommended designers to use common region in real contexts to communicate complex informational configurations. Our results could have some implications for the communicative applications of visualization by providing some cues that improve the organization of information in charts, matrices or lists. Furthermore, the indirect effects of extrinsic grouping cues might be useful for improving object perception in patients with visual system damage (Gilleber \& Humphreys, 2015).

Interestingly, the unequivocal processing dominance of common region in our study occurred even though the phenomenological grouping strength of the grouping cues was equated for each participant. The absence of correlation between the data from the scaling task and the performance in the RDT reinforces the dissociation between both measures. Remarkably, the inspection of individual data revealed a processing advantage for common region in the competing conditions even for the only participant who showed faster RTs to connectedness compared to common region in the single grouped conditions (see supplementary materials: section b). Finally, the finding of similar RT differences in both competing and single conditions in a preliminary study with scaling tasks in which common region was conveyed by luminance provides further evidence for the consistency of our data (see supplementary materials: section a).

In fact, these results were not completely unexpected since similar discrepancies between direct and indirect measures of grouping have been repeatedly observed in previous studies (Schmidt and Schmidt 2013; Luna et al., 2016; Montoro et al., 2015; Palmer and Beck, 2007). This dissociation has been attributed to the fact that the perceptual parameters of grouping are not necessarily represented in an equivalent manner by the phenomenological perception and by the visuo-motor system. Consequently, equating two stimuli with a subjective method does not guarantee a parallel effect in the visuo- 
motor system. This finding is especially relevant because it uncovers the dissociated cognitive mechanisms involved in perceptual grouping tasks. Additionally, it leads directly to an important new question, which should be addressed in future research, that is: will visual parameters generating identical behavioral effects be perceived as similar by phenomenological experience? (See Schmidt \& Schmidt, 2013, for a similar account.)

As other authors have discussed, none of the measures should be privileged a priori to the detriment of the other because they both have strengths and weaknesses and they are convergent measures of grouping (Kubovy \& Gepshtein, 2003; Palmer \& Beck, 2007). In phenomenological methods, a direct measure of grouping is achieved based on participant reports of the grouping strength they perceive in each trial, so a more cognitive than perceptual evaluation strategy might be adopted. In contrast, the removal of spontaneous grouping in indirect tasks leaves open the possibility of not being able to measure purely grouping (Kubovy \& Gepshtein, 2003; Palmer \& Beck, 2007). Notably, as in the current study, it seems crucial to avoid the adoption of alternative strategies non-related to grouping operations when exploring extrinsic grouping. Nonetheless, a complete characterization of the dominance dynamics of grouping needs convergent evidence with different types of measures.

Author note This work was supported by the Ministerio de Economía y Competitividad (MINECO) of Spain (PSI2015-68368-P (MINECO/ FEDER)) and the Comunidad de Madrid (H2015/HUM-3327). The authors acknowledge two reviewers for their insightful comments on a previous version of the manuscript.

\section{References}

Bae, J., \& Watson, B. (2014). Reinforcing visual grouping cues to communicate complex informational structure. IEEE Trans Vis Comput Graph, 20, 1973-1982.

Brooks, J. L. (2015). Traditional and new principles of perceptual grouping. In J. Wagemans (Ed.), Ocxford handbook of perceptual organization (pp. 57-87). Oxford: Oxford University Press.

Gillebert, C. R., \& Humphreys, G. (2015). Mutual interplay between perceptual organization and attention. In J. Wagemans (Ed.), The oxford handbook of perceptual organization. Oxford: Oxford University Press.
Kubovy, M., \& Gepshtein, S. (2003). Perceptual grouping in space and in space-time: An exercise in phenomenological psychophysics. In M. Behrmann, R. Kimchi, \& C. R. Olson (Eds.), Perceptual organization in vision: Behavioral and neural perspectives (pp. 45-85). Mahwah: Lawrence Erlbaum.

Kubovy, M., \& van den Berg, M. (2008). The whole is equal to the sum of its parts: A probabilistic model of grouping by proximity and similarity in regular patterns. Psychol Rev, $115,131-154$.

Luna, D., \& Montoro, P. R. (2011). Interactions between intrinsic principles of similarity and proximity and extrinsic principle of common region in visual perception. Perception, 40, 1467-1477.

Luna, D., Montoro, P. R., Villalba-García, C., \& Hinojosa, J. A. (2016). Dominance dynamics of competition between intrinsic and extrinsic grouping cues. Acta Psychol, 170, 146-154.

Montoro, P. R., \& Luna, D. (2015a). Competición y cooperación entre principios intrínsecos y extrínsecos de agrupamiento perceptivo en el ámbito de la visión. Anales de Psicología, 31, 345-354.

Montoro, P. R., \& Luna, D. (2015b). Does the relative strength of visual grouping principles modulate cooperation and competition between them? A study of grouping by proximity and common region. Span $J$ Psychol, 18, 1-10.

Montoro, P. R., Luna, D., Albert, J., Santaniello, G., López-Martín, S., Pozo, M. A., \& Hinojosa, J. A. (2015). A temporo-spatial analysis of the neural correlates of extrinsic perceptual grouping in vision. Neuropsychologia, 69, 118-129.

Navon, D. (1977). Forest before trees: The precedence of global features in visual perception. Cogn Psychol, 9, 353-383.

Palmer, S. E. (1992). Common region: A new principle of perceptual grouping. Cogn Psychol, 24, 433-447.

Palmer, S. E. (1999). Vision science. Photons to phenomenology. Cambridge: MIT Press.

Palmer, S. E., \& Beck, D. (2007). The repetition discrimination task: An objective method for studying perceptual grouping. Perception and Psychophysics, 69, 68-78.

Palmer, S. E., \& Rock, I. (1994). Rethinking perceptual organization: The role of uniform connectedness. Psychon Bull Rev, 1, 29-55.

Peterson, M., \& Kimchi, R. (2015). Perceptual organization in vision. In D. Reisberg (Ed.), Oxford handbook of cognitive psychology (pp. 931). New York: Oxford University Press.

Quinlan, P. T., \& Wilton, R. L. (1998). Grouping by proximity or similarity? Competition between the Gestalt principles in vision. Perception, 27, 417-430.

Quinn, P. C., \& Bhatt, R. S. (2015). Development of perceptual organization in infancy. In J. Wagemans (Ed.), Oxford handbook of perceptual organization (pp. 691-712). Oxford: Oxford University Press.

Schmidt, F., \& Schmidt, T. (2013). Grouping principles in direct competition. Vis Res, 88, 9-21.

Wertheimer, M. (1923). Untersuchungen zur Lehre von der Gestalt II. Psycholosgische Forschung, 4, 301-350. 